\title{
Usefulness of measuring bone density of mandibular condyle in patients at risk of osteoporosis: A cone beam computed tomography study
}

\author{
Mustafa Alkhader ${ }^{1}$, Ammar Aldawoodyeh $^{2}$, Nour Abdo $^{3}$
}

Correspondence: Dr. Mustafa Alkhader

Email:mmalkhader@just.edu.jo

\begin{abstract}
'Department of Oral Surgery and Medicine, Faculty of Dentistry, Jordan University of Science and Technology, Irbid, Jordan,

2Department of Preventive Dentistry, Faculty of Dentistry, Jordan University of Science and Technology, Irbid, Jordan,

${ }^{3}$ Department of Community Medicine, Public Health and Family Medicine, Faculty of Medicine, Jordan University of Science and Technology, Irbid, Jordan
\end{abstract}

\section{ABSTRACT}

Objective: Using cone beam computed tomography (CBCT) images, the aim of the study was to evaluate the usefulness of measuring bone density of mandibular condyle (BDMC) in patients at risk of osteoporosis. Materials and Methods: Two hundred and four mandibular condyles ( 46 condyles from males and 158 condyles from females) were examined by CBCT. Using inVivo software (Anatomage, San Jose, Calif), BDMC and mental index (MI) were measured by two observers and correlated using Pearson's correlation coefficients. Patients were divided into high risk and low risk of osteoporosis using $3.1 \mathrm{~mm}$ of MI as a cutoff value, and the mean value of BDMC was compared and correlated using independent samples $t$-test and regression analysis. Receiver-operating characteristic (ROC) curve analyses were also used to examine the predictive power of BDMC. Results: The mean value of BDMC was moderately correlated with MI $(r=0.38)$, and in female patients at low risk of osteoporosis, the mean value of BDMC was significantly higher than in patients at high risk of osteoporosis. In multivariate linear regression analysis, being at risk of osteoporosis is significantly affected by BDMC, age, and gender. ROC analysis showed that the mean value of BDMC had a moderate predictive power for predicting patients at risk of osteoporosis (area under the curve $=0.621$ for females and 0.649 for males). Conclusions: Measuring BDMC is considered useful in predicting patients at risk of osteoporosis.

Key words: Bone density, cone beam computed tomography, mandibular condyle, osteoporosis

\section{INTRODUCTION}

Osteoporosis defined as "a disease characterized by low bone mass and microarchitectural deterioration of bone tissue, leading to enhanced bone fragility and a consequent increase in fracture risk." ${ }^{[1]}$ Complications

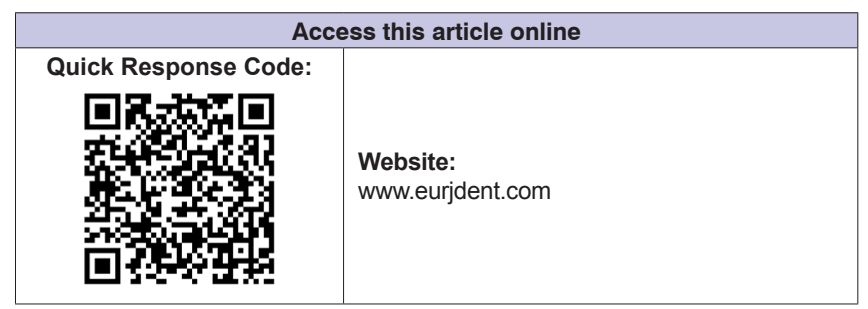

of osteoporosis such as osteoporotic fracture are associated with high morbidity and medical costs. The risk for such complications can be reduced if the

This is an open access journal, and articles are distributed under the terms of the Creative Commons Attribution-NonCommercial-ShareAlike 4.0 License, which allows others to remix, tweak, and build upon the work non-commercially, as long as appropriate credit is given and the new creations are licensed under the identical terms.

For reprints contact: reprints@medknow.com

How to cite this article: Alkhader $\mathrm{M}$, Aldawoodyeh $\mathrm{A}$, Abdo $\mathrm{N}$ Usefulness of measuring bone density of mandibular condyle in patients at risk of osteoporosis: A cone beam computed tomography study. Eur J Dent 2018;12:363-8.

DOI: 10.4103/ejd.ejd_272_17 
disease is diagnosed and treated earlier, and this is where the importance of finding new screening tools for osteoporosis comes from.

The current gold standard for diagnosing osteoporosis is dual-energy X-ray absorptiometry (DEXA) or DEXA, which is a test that is used to measure bone mineral density (BMD) ${ }^{[2]}$ Although DEXA is used extensively around the world because of its reliability and low radiation dose, it is not always available and is not considered as cost-effective tool for screening in low-risk patients, such as younger individuals. ${ }^{[2]}$ Quantitative computed tomography (CT) can be used instead of DEXA to diagnose osteoporosis, ${ }^{[3]}$ but it is also expensive and associated with higher radiation dose, thus would not be appropriate as screening tool too.

In an effort to increase dentists role in screening potential osteoporosis patients, and to increase the likelihood that these patient are discovered earlier in life, many indices related to panoramic radiograph have been proposed. ${ }^{[4]}$ These indices can be used to analyze mandibular bone by two ways; either by objective analysis, in which linear measurements are calculated, or by subjective analysis, in which the appearance of the bone is described according to a

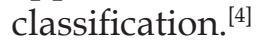

The commonly used method for subjective analysis is Klemetti index (KI), which classifies the inferior mandibular cortex appearance to three categories: $\mathrm{C} 1$ - the endosteal margin of the cortex is even and sharp on both sides; $\mathrm{C} 2$ - the end osteal margin shows semilunar defects and/or seems to form endosteal cortical residues on one or both sides; and C3 - the cortical layer forms heavy endosteal cortical residues and is clearly porous. ${ }^{[5]}$ Although many studies were conducted and suggested that this index can be used as a screening tool, Calciolari et al. ${ }^{[4]}$ reported that the wide range of outcomes is a sign of high heterogeneity between these studies, this may be attributed to the subjectivity of this index. In fact, in a more recent study, this index was reported to be of low sensitivity and specificity, and concluded that it is of limited use in clinical practice. ${ }^{[6]}$

On the other hand, other morphometric analysis dependent indices have been described; the most famous of them are panoramic mandibular index (PMI) and mental index (MI). The PMI index is defined as the ratio of the thickness of the inferior mandibular cortex in the mental region over the distance between the lower border of the mandible and either the inferior or the superior border of the mental foramen. ${ }^{[4]}$ Some studies showed significant correlation between PMI index and osteoporosis, but others did not. ${ }^{[7-10]}$ Furthermore, Horner and Devlin showed that the PMI has no significant advantage over the MI. ${ }^{[1]}$

The MI (also known as mandibular cortical width or mandibular cortical thickness) is measured at the mental foramen region, along a line passing through the middle of the mental foramen and perpendicular to the tangent to the lower border of the mandible. ${ }^{[4]} \mathrm{MI}$ is considered the most established among mandibular cortical indices. ${ }^{[12]}$ Other indices, such as the KI, the gonial, and antegonial indices, were less frequently used and are less established.

Another more recent imaging modality, cone beam $\mathrm{CT}(\mathrm{CBCT})$, is being widely used in dentistry. Koh and $\mathrm{Kim}^{[13]}$ were the first to examine the potential use of CT indices on CBCT images for assessment of BMD. Mostafa et al..$^{[14]}$ found a significant correlation between CT mental index (CTMI) scores and osteoporosis.

$\mathrm{CBCT}$ is considered the standard of care for imaging osseous structures of the temporomandibular joint (TMJ), mainly because it has better imaging quality and less radiation dose compared to helical CT. ${ }^{[15]}$ More recent studies suggest using an alternative approach that utilizes two CBCT acquisitions of the right and left TMJs, as this results in $>10$-fold reduction in the radiation do when compared to full field CBCT. ${ }^{[16]}$

As mentioned above, for osseous assessment of TMJ, CBCT images from the small field of view are recommended. However, in such case, the mandible will not be present, and it will not be possible to predict osteoporosis using mandibular cortical indices. Therefore, to enhance dentists' role in screening patients for osteoporosis, and to maximize patients' benefits from getting such images, we find it necessary to know if these images can be used to screen patients for osteoporosis or not.

Kosugi et al. ${ }^{[17]}$ were the first to study the effect of osteoporosis on bone density of mandibular condyle (BDMC) in rats, and they found that the BDMC is less in osteoporotic rats compared to normal rats when measured using morphometric analysis indices for micro-CT scans. To the best of our knowledge, no one has ever studied the relationship between BDMC and osteoporosis in humans; therefore this study was conducted. 


\section{MATERIALS AND METHODS}

\section{Patients}

In our retrospective study, CBCT images for all patients aged 50 years and more were retrieved and evaluated from our CBCT database. All patients underwent CBCT examination for dental implant treatment and other dental purposes at our dental radiology clinic between January 2011 and January 2017. After excluding cases with artifacts or pathology affecting measurements of BDMC or MI, the number of cases included was 204 mandibular condyles (46 condyles from males and 158 condyles from females). Patients aged between 50 and 78 years (mean age $59.75 \pm 7.01$ ). The present study is part of a protocol which was approved by the Local Ethical Committee with the protocol number 447/2016.

\section{Cone beam computed tomography examination}

As a CBCT apparatus, KODAK 9500 Cone Beam 3D System (Carestream, Rochester, NY, USA) with flat panel detector was used. The imaging area of CBCT is a cylinder with a height of $15-20.6 \mathrm{~cm}$ and a diameter of 9-18 cm providing isotropic cubic voxels with sides approximating $0.2-0.3 \mathrm{~mm}$. Only cases examined with $0.2 \mathrm{~mm}$ of voxel size were included in the study. The exposure parameters were: $90 \mathrm{kV}$ as a tube voltage, $10 \mathrm{~mA}$ as a tube current, and $10.8 \mathrm{~s}$ as an exposure time.

Examinations were performed by $360^{\circ}$ rotation in the occlusal position with the patient standing and closing their teeth.

\section{Images}

Two calibrated observers used inVivo software (Anatomage, San Jose, Calif) for measuring BDMC and MI.

For measuring BDMC, a coronal section in the middle of each mandibular condyle was used in arch section module. To obtain the coronal section in the middle of mandibular condyle, the middle of mandibular condyle was localized on reconstructed panoramic image using the associated vertical colored bar. A circle touching inner cortical surfaces was drawn using circle notation tool, and then using the distance measurement tool, $6 \mathrm{~mm} \times 6 \mathrm{~mm}$ square area of interest was drawn inside the circle starting from top to the bottom. Finally, Hounsfield Units [HUs] measurement tool was used to generate density value for the corresponding condyle. HU measurement tool showed 3 density values at each condyle (minimum, mean, and maximum).
However, we only considered the mean density value for analysis [Figure 1a].

For measuring MI (inferior cortical width of the mandible at mental foramen), arch section module was utilized, and a cross-sectional image in the middle of each mental foramen was used. To obtain a cross-sectional image at the middle of mental foramen, the middle of mental foramen was localized on corresponding panoramic image using associated vertical colored bar, then using the distance measurement tool, the inferior cortical width of the mandible was measured at each mental foramen following the example of Koh and $\mathrm{Kim}^{[13]}$ [Figure 1b].

All images were evaluated on a high definition LCD display, and window settings were fixed for all cases.

\section{Statistical analysis}

Data were analyzed using SAS 9.2 (SAS Institute, Cary, NC). Data were described using means and percentages. Pearson's correlation coefficient was used to determine inter-rater correlation between first and second observer measurements of BDMC and $\mathrm{MI}$, and the correlation between BDMC and MI. According to a multi-center study with large sample size, ${ }^{[18]}$ patients with the thinnest MI $(\leq 3 \mathrm{~mm})$ have the highest likelihood of osteoporosis. Therefore, using $3.1 \mathrm{~mm}$ of $\mathrm{MI}$ as a cutoff value, patients were divided into low and high risk of osteoporosis. Independent $t$-test was used to compare means of continuous variables between those with low and high risk of osteoporosis. The association between BDMC and

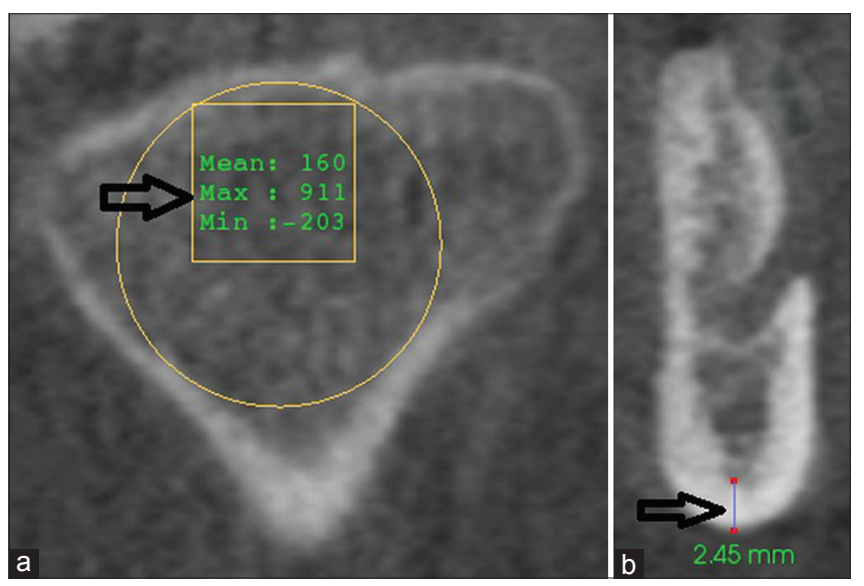

Figure 1: A 68-year-old female patient who visited our dental clinic for implant placement (a) Cross-sectional cone beam computed tomography image at the middle of right mandibular condyle showing bone density values of the square area of interest (arrow). (b) Cross-sectional cone beam computed tomography image at the middle of right mental foramen showing inferior cortical width (mental index) (arrow) 
MI after adjusting for age and gender were tested in linear regression analysis when $\mathrm{MI}$ is continuous and binary logistic regression when MI is dichotomous. Receiver operating characteristics (ROCs) analysis was used to test the ability of BDMC to predict the risk of osteoporosis. $P<0.05$ was considered statistically significant.

\section{RESULTS}

This study included a total of 204 mandibular condyles (46 condyles from males and 158 condyles from females). The intra-rater correlations for BDMC $(r=0.99)$ and MI $(r=0.98)$ were very strong. Therefore, the two readings for each measurement were averaged to be used for further analysis. The correlation between average BDMC and average MI was moderate $(r=0.32)$.

Males had significantly higher mean of MI [Table 1]. Although males had higher BDMC mean compared to females, it was not statistically significant [Table 1]. Patients were divided into high risk and low risk of osteoporosis using $3.1 \mathrm{~mm}$ of MI as a cutoff value, and females had significantly higher risk than males for osteoporosis [Table 2]. For females only, the mean age was significantly higher for females at high risk of osteoporosis compared to females at low risk of osteoporosis (61.99 [7.6] year vs. 57.38 [4.6] year; $P<0.0001$ ) [Table 3]. Furthermore, females at high risk of osteoporosis have lower BDMC compared to females at low risk of osteoporosis (206 [102.14] vs. 236.21 [83.72] year; $P<0.046)$ [Table 3]. After adjusting for the effects of age and gender, the mean value of $\operatorname{BDMC}(F=10.87$, $P<0.0001)$ remained significantly higher in patients at

\begin{tabular}{|c|c|c|c|}
\hline & Males $(n=46)$ & Females $(n=158)$ & $P$ \\
\hline & Mean (range) $\pm S D$ & Mean (range) \pm SD & \\
\hline Mental index & $3.51(1.65-5.30) 0.91$ & $3.12(1.29-7.25) 1.01$ & $0.0197^{*}$ \\
\hline Density & $\begin{array}{c}245.16(83.50-512.0) \\
108.51\end{array}$ & $\begin{array}{c}221.16(37.5-470.5) \\
94.3\end{array}$ & 0.1439 \\
\hline
\end{tabular}

$P$ values were determined by $t$-test. SD: Standard deviation

\begin{tabular}{|c|c|c|c|}
\hline Osteoporosis risk & High risk (\%) & Low risk (\%) & $P$ \\
\hline \multicolumn{4}{|l|}{ Gender } \\
\hline Male & $13(28.3)$ & $33(71.7)$ & $0.0059^{*}$ \\
\hline Female & $81(51.3)$ & $77(48.7)$ & \\
\hline
\end{tabular}

${ }^{*}$ Females have higher risk than males for osteoporosis; $P$ values were determined by Chi-square test low risk of osteoporosis. ROC analysis showed that the mean value of BDMC (area under the curve $=0.621$ for females and 0.649 for males) had a moderate predictive power to predict osteoporosis.

\section{DISCUSSION}

In this study, we evaluated the usefulness of BDMC in predicting osteoporosis in patients aged 50 years old and more. According to Jonasson et al. ${ }^{[19]}$ mandibular cortical thickness decreased significantly after the age of 50, and more fractures were shown. Therefore, patients above 50 years old are at risk of osteoporosis and possibility to have low MI is high as shown in similar studies. ${ }^{[6,20]}$

As we mentioned in the introduction, a relationship between the density of the condyle in mico-CT images and osteoporosis was established in in vitro studies. ${ }^{[17]}$ On the other hand, Güngör et al. ${ }^{[21]}$ showed that a positive correlation is also present between femoral head BMD and left condyle density value in CBCT images. According to our results, the difference in BDMC between high-risk and low-risk osteoporosis group is statistically significant in females. This suggests that the osteoporosis can affect the BDMC in human too.

It is worth mentioning that $\mathrm{CT}$ and $\mathrm{CBCT}$ images of other parts of the mandible have already been found to be useful for the same purpose. For example, Barngkgei et al. ${ }^{[22]}$ concluded in their study that the radiographic density of the body of the mandible in CBCT images can be used to accurately predict lumber vertebrate and femoral neck osteoporosis. Others, such as Mostafa et al. ${ }^{[14]}$ found that CT cortical index scores, CTMI, and computed tomography mandibular index (CTI) can be used to differentiate between the control and the osteoporotic groups in postmenopausal women.

In the previous studies, which measured MI on cross-sectional CBCT images, ${ }^{[14,21]}$ MI was significantly higher in patients at low risk of osteoporosis. Our results are also in agreement with these studies.

In accordance with our results, several studies showed that MI has higher values in males and inversely related to age. ${ }^{[2,23-25]}$ Nevertheless, in our study the mean age was significantly higher for females at high risk of osteoporosis compared to females at low risk of osteoporosis; this could be due to the possibility of having more females at menopause or postmenopause at older age. 
Alkhader, et al.: Bone density of mandibular condyle in patients at risk of osteoporosis

\begin{tabular}{|c|c|c|c|c|c|c|}
\hline \multirow[t]{2}{*}{ Gender } & \multirow{2}{*}{$\begin{array}{l}\text { Parameter } \\
(n)\end{array}$} & \multicolumn{2}{|c|}{ High risk of osteoporosis } & \multicolumn{2}{|c|}{ Low risk of osteoporosis } & \multirow[t]{2}{*}{$P$} \\
\hline & & Mean \pm SD & $n$ & Mean $\pm S D$ & $n$ & \\
\hline \multirow[t]{3}{*}{ Females } & $\mathrm{Ml}$ & $2.37(0.73)$ & 79 & $3.88(0.61)$ & 79 & $<0.0001$ \\
\hline & Density & $206.12(102.14)$ & 79 & $236.21(83.72)$ & 79 & 0.0446 \\
\hline & Age & $61.99(7.57)$ & 79 & $57.38(4.85)$ & 79 & $<0.0001$ \\
\hline \multirow[t]{3}{*}{ Males } & $\mathrm{Ml}$ & $2.42(0.56)$ & 14 & $3.99(0.53)$ & 32 & $<0.0001$ \\
\hline & Density & $219.86(82.22)$ & 14 & $256.23(117.64)$ & 32 & 0.3007 \\
\hline & Age & $61.36(9.60)$ & 14 & $59.34(7.16)$ & 32 & 0.4340 \\
\hline
\end{tabular}

With regard to the dentition status, both of Xu et al. ${ }^{[26]}$ and Aggarwal et al. ${ }^{[27]}$ found significant difference between right and left condyle bone density in patients with unilateral loss of teeth, with the density being lower in the edentulous half. Choi et al. ${ }^{[28]}$ reported on the same issue, but his results specified the presence of molars to be the important factor affecting condyle density. Despite being significant factor, the dentition status remained outside the scope of our study. In the same study of Choi et al., ${ }^{[28]}$ they found that age was affecting factor when it comes to condyle density in both males and females as measured from micro-CT scan. In our study, the age was a significant factor for females only. This may be explained by the fact that the age range in our study included only old patients, while wider age range including younger individuals was selected for the previously mentioned study.

In a study done by Yamada et al., ${ }^{[29]}$ a correlation between bone density of lumbar spine (L1-L3) and BDMC was present, and both decrease with age in a similar fashion to each other. Their data were obtained using quantitative CT; similarly, our data obtained using CBCT, which is being used by dentists for different dental applications.

Both of the studies support the idea that the BDMC can be used to predict patients at risk of osteoporosis. Although dual X-ray absorptiometry (DXA) as the gold standard for diagnosis of osteoporosis was not utilized in both of the studies, DXA has several limitations especially in obese patients and/or patients with degenerative changes in the spine. ${ }^{[3]}$ Moreover, DXA is not available in routine dental practice as mentioned previously.

With regards to the usefulness of BDMC in predicting patients at high risk of osteoporosis, the area under the ROC curve was 0.621 for females and 0.649 for males. This indicates moderate predictive power of BDMC. Similar results were obtained by using
MI measurements for predicting osteoporosis. ${ }^{[12,20]}$ Therefore, BDMC might be recommended to be used instead of MI when mandibular bone is not shown in patient images for any reason.

In this study, all patients with $\mathrm{MI} \leq 3 \mathrm{~mm}$ were considered at high risk of osteoporosis as shown in previous studies, ${ }^{[6,18,30]}$ however, the cutoff value of MI might be related to race or ethnic group. ${ }^{[20]}$ We could not test the accuracy of the previous cutoff value; this is considered a limitation of this study. Moreover, the dimensions of area of interest used in this study might not be applicable on images of patients from different ethnic group.

\section{CONCLUSIONS}

Taking into account the limitations of the present study, our results indicate that measuring BDMC is considered useful in predicting patients at risk of osteoporosis.

\section{Financial support and sponsorship \\ Nil.}

\section{Conflicts of interest}

There are no conflicts of interest.

\section{REFERENCES}

1. Nakamoto T, Taguchi A, Ohtsuka M, Suei Y, Fujita M, Tanimoto K, et al. Dental panoramic radiograph as a tool to detect postmenopausal women with low bone mineral density: Untrained general dental practitioners' diagnostic performance. Osteoporos Int 2003;14:659-64

2. Morgan SL, Prater GL. Quality in dual-energy X-ray absorptiometry scans. Bone 2017;104:13-28.

3. Link TM, Lang TF. Axial QCT: Clinical applications and new developments. J Clin Densitom 2014;17:438-48.

4. Calciolari E, Donos N, Park JC, Petrie A, Mardas N. Panoramic measures for oral bone mass in detecting osteoporosis: A systematic review and meta-analysis. J Dent Res 2015;94:17S-27S.

5. Klemetti E, Kolmakov S, Kröger H. Pantomography in assessment of the osteoporosis risk group. Scand J Dent Res 1994;102:68-72.

6. Jagelaviciene E, Krasauskiene A, Zalinkevicius R, Kubilius R, Vaitkeviciene I. The relationship between the calcaneal bone mineral density and the mental index in post-menopausal females. 


\section{Alkhader, et al.: Bone density of mandibular condyle in patients at risk of osteoporosis}

Dentomaxillofac Radiol 2013;42:20120050.

7. Drozdzowska B, Pluskiewicz W, Tarnawska B. Panoramic-based mandibular indices in relation to mandibular bone mineral density and skeletal status assessed by dual energy X-ray absorptiometry and quantitative ultrasound. Dentomaxillofac Radiol 2002;31:361-7.

8. Mohajery M, Brooks SL. Oral radiographs in the detection of early signs of osteoporosis. Oral Surg Oral Med Oral Pathol 1992;73:112-7.

9. Watson EL, Katz RV, Adelezzi R, Gift HC, Dunn SM. The measurement of mandibular cortical bone height in osteoporotic vs. non-osteoporotic postmenopausal women. Spec Care Dentist 1995;15:124-8.

10. Marandi S, Bagherpour A, Imanimoghaddam M, Hatef M, Haghighi A. Panoramic-based mandibular indices and bone mineral density of femoral neck and lumbar vertebrae in women. J Dent (Tehran) 2010;7:98-106.

11. Horner K, Devlin H. The relationship between mandibular bone mineral density and panoramic radiographic measurements. J Dent 1998;26:337-43.

12. Leite AF, Figueiredo PT, Guia CM, Melo NS, de Paula AP. Correlations between seven panoramic radiomorphometric indices and bone mineral density in postmenopausal women. Oral Surg Oral Med Oral Pathol Oral Radiol Endod 2010;109:449-56.

13. Koh KJ, Kim KA. Utility of the computed tomography indices on cone beam computed tomography images in the diagnosis of osteoporosis in women. Imaging Sci Dent 2011;41:101-6.

14. Mostafa RA, Arnout EA, Abo El-Fotouh MM. Feasibility of cone beam computed tomography radiomorphometric analysis and fractal dimension in assessment of postmenopausal osteoporosis in correlation with dual X-ray absorptiometry. Dentomaxillofac Radiol 2016;45:20160212.

15. Karatas $\mathrm{OH}$, Toy E. Three-dimensional imaging techniques: A literature review. Eur J Dent 2014;8:132-40.

16. Lukat TD, Wong JC, Lam EW. Small field of view cone beam CT temporomandibular joint imaging dosimetry. Dentomaxillofac Radiol 2013;42:20130082.

17. Kosugi K, Yonezu H, Kawashima S, Honda K, Arai Y, Shibahara T, et al. A longitudinal study of the effect of experimental osteoporosis on bone trabecular structure in the rat mandibular condyle. Cranio 2013;31:140-50.

18. Devlin H, Karayianni K, Mitsea A, Jacobs R, Lindh C, van der Stelt P, et al. Diagnosing osteoporosis by using dental panoramic radiographs: The OSTEODENT project. Oral Surg Oral Med Oral Pathol Oral Radiol Endod 2007;104:821-8.
19. Jonasson G, Sundh V, Hakeberg M, Hassani-Nejad A, Lissner L, Ahlqwist $\mathrm{M}$, et al. Mandibular bone changes in 24 years and skeletal fracture prediction. Clin Oral Investig 2013;17:565-72.

20. Kim OS, Shin MH, Song IH, Lim IG, Yoon SJ, Kim OJ, et al. Digital panoramic radiographs are useful for diagnosis of osteoporosis in Korean postmenopausal women. Gerodontology 2016;33:185-92.

21. Güngör E, Yildirim D, Çevik R. Evaluation of osteoporosis in jaw bones using cone beam CT and dual-energy X-ray absorptiometry. J Oral Sci 2016;58:185-94.

22. Barngkgei I, Al Haffar I, Khattab R. Osteoporosis prediction from the mandible using cone-beam computed tomography. Imaging Sci Dent 2014;44:263-71.

23. Dagistan S, Bilge OM. Comparison of antegonial index, mental index, panoramic mandibular index and mandibular cortical index values in the panoramic radiographs of normal males and male patients with osteoporosis. Dentomaxillofac Radiol 2010;39:290-4.

24. Govindraju P, Chandra P. Radiomorphometric indices of the mandible-An indicator of osteoporosis. J Clin Diagn Res 2014;8:195-8.

25. Bajoria AA, Ml A, Kamath G, Babshet M, Patil P, Sukhija P, et al. Evaluation of radiomorphometric indices in panoramic radiograph $-\mathrm{A}$ screening tool. Open Dent J 2015;9:303-10.

26. $\mathrm{Xu} \mathrm{W}, \mathrm{Lu} \mathrm{H}$, Shi Q, Gu Z. Research on condylar morphology in patients with prolonged unilateral posterior teeth loss with cone beam computed tomography. Hua Xi Kou Qiang Yi Xue Za Zhi 2016;34:162-5.

27. Aggarwal H, Singh RD, Kumar M, Singh R, Siddhartha R, Jurel SK, et al. Three-dimensional quantitative analysis of the bone density of mandibular condyle in dentulous and edentulous jaws: An in vivo study. J Clin Densitom 2015;18:50-3.

28. Choi DY, Sun KH, Won SY, Lee JG, Hu KS, Kim KD, et al. Trabecular bone ratio of the mandibular condyle according to the presence of teeth: A micro-CT study. Surg Radiol Anat 2012;34:519-26.

29. Yamada M, Ito M, Hayashi K, Sato H, Nakamura T. Mandibular condyle bone mineral density measurement by quantitative computed tomography: A gender-related difference in correlation to spinal bone mineral density. Bone 1997;21:441-5.

30. Gulsahi A, Yüzügüllü B, Imirzalioglu P, Genç Y. Assessment of panoramic radiomorphometric indices in Turkish patients of different age groups, gender and dental status. Dentomaxillofac Radiol 2008;37:288-92. 